\title{
BEHAVIOR OF FISH REEF BLOCKS FALLING THROUGH WATER
}

\author{
Jun Mitsui ${ }^{1}$, Shin-ichi Ogata ${ }^{1}$, Sanae Shibata ${ }^{1}$, Akira Matsumoto $^{1}$ and Masato Yamamoto ${ }^{2}$
}

\begin{abstract}
An accurate and economical placement method for fish reef blocks utilizing free fall in the deep sea area is presented in this paper. Falling behavior of concrete blocks in water was investigated by hydraulic model experiments and 3-D numerical computations. As a result, a new fish reef block which falls through water with highly stable behavior was developed. The distribution range of the developed block on the sea bottom was predicted by a stochastic model and it was proved to be sufficiently narrow. In addition, a quick estimation method for the placement position was developed to determine the release point of the block in actual construction. By using hydrodynamic force coefficients to evaluate the forces acting on the block, the method can estimate the falling behavior of the block in a flow field with sufficient accuracy and in a short time.
\end{abstract}

Keywords: fish reef block; snow crab; falling behavior; deep sea area; hydrodynamic force coefficient; OpenFOAM

\section{INTRODUCTION}

Snow crabs are an important fishery resource in Japan. The snow crab catch in the western part of the Sea of Japan decreased sharply from around 1965 through the late 1970s due to overfishing. In recent years it is steadily recovering thanks to catch limits (Moriyama, 2011). For the purpose of a more reliable recovery to a high-level amount of resource, the Fisheries Agency of Japan has been construct nursery reefs for snow crabs in the last eight years since 2007. The nursery reefs are constructed placing concrete blocks at intervals of about $250 \mathrm{~m}$ in an area $2 \mathrm{kms}$ square. The water depth of the construction area is about $250 \mathrm{~m}$ which is the habitation depth of female snow crabs.

Generally, the concrete blocks are placed on the sea bottom using wire ropes (Fig. 1(a)). However, this method is uneconomical in the case of deep water because it takes a long time to anchor a floating crane and install the blocks in such deep water. On the other hand, though a method by free fall (Fig. 1(b)) is more economical, the accuracy of installation of the blocks becomes a problem. The accuracy requested for the placement position is a range within $30 \mathrm{~m}$ in radius around the planned position. To make this method possible, the following two conditions must be met.

- The block must be a shape which falls with stable behavior in water.

- The placement position of the block in a flow field must be predictable to decide the release point.

In this study, a new fish reef block which falls with stable behavior was developed by hydraulic model experiments and 3-D numerical computations. At first a fundamental shape of the block was decided from the results of hydraulic model experiments. Then the shape was improved by using numerical analysis. The probability distribution of the developed block on the sea bottom was predicted by stochastic model. It was confirmed that the distribution range of the placement position satisfies the requested one. In the rest of this paper, a quick estimation method of the placement position is described. In the method, hydrodynamic force coefficients calculated beforehand were used to evaluate the forces acting on the block to reduce the computation time. The method was validated by comparison with experimental results in a flow field.

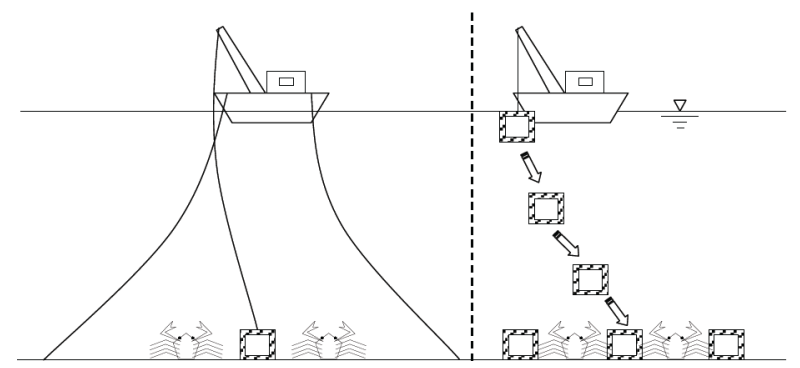

(a) Conventional method

(b) New method

Figure 1. Comparison of construction methods.

\footnotetext{
${ }^{1}$ Technical Research Institute, Fudo Tetra Corporation, 2-7, Higashi-nakanuki, Tsuchiura, Ibaraki, 300-0006, Japan

${ }^{2}$ Tripole Co.,Ltd., Iron Quarter Building 5F, 1-1-6, Saga, Koto-ku, Tokyo, 135-0031, Japan.
} 


\section{DEVELOPMENT OF A NEW FISH REEF BLOCK}

\section{Hydraulic Model Experiments}

Hydraulic model experiments were conducted to decide a fundamental shape of a block which would fall with stable behavior in water. Fig. 2 shows the experimental setup. The model scale is $1 / 60$. A water tank made of acrylic of size $1.0 \mathrm{~m}$ square and $2.3 \mathrm{~m}$ high was used. The water depth was $2.0 \mathrm{~m}$ ( $120 \mathrm{~m}$ in prototype scale). The model block made of mortar was released gently from just under the water surface. The behavior of the block was recorded with two video cameras from two directions orthogonal to each other. The position of the block at each moment was obtained from the video image.

Several kinds of model blocks with frame structures were tested, for example cubic, prismoid and circular cylinder, etc. The cubic type shown in Fig. 3(a) (hereafter the "Prototype block") was selected as it fell without rotation and the amplitude of rolling motion was smallest among them. The block was improved by numerical analysis as mentioned below and shown in Fig. 3(b) (hereafter the "Improved block") was also tested. The tests with the "Improved block" were repeated about 100 times to investigate the probabilistic distribution of the placement position.

As the falling behavior is influenced by current fields in actual construction, experiments in a flow field were also conducted. A submersible pump was installed in the flume to generate a steady flow. The water depth was about $0.9 \mathrm{~m}(54 \mathrm{~m}$ in prototype scale) and the flow velocity about $5 \mathrm{~cm} / \mathrm{s}(0.39 \mathrm{~m} / \mathrm{s}$ in prototype scale) and was vertically uniform. The experiments in the flow fields were conducted with the "Improved block".

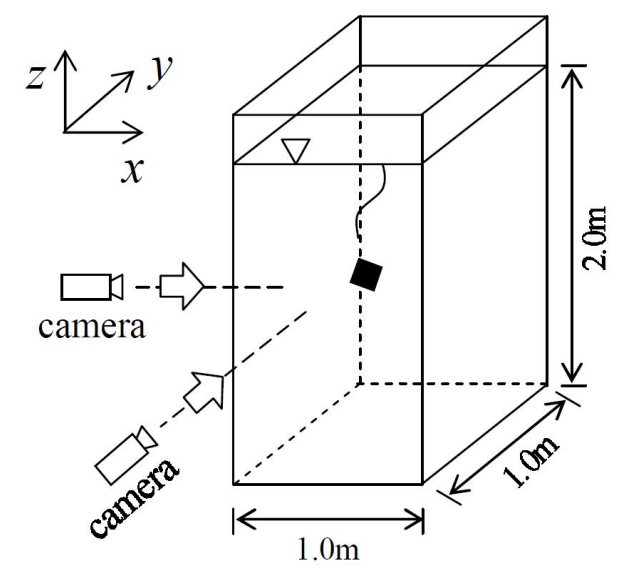

Figure 2. Experimental setup.

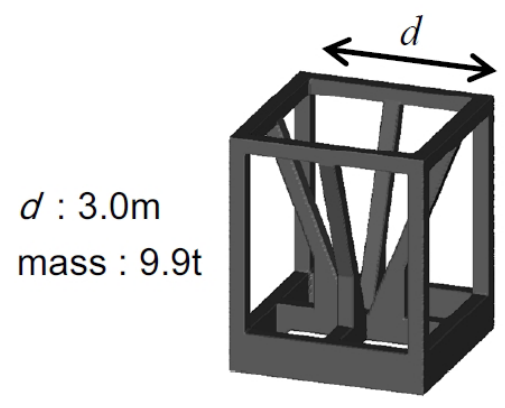

(a) Prototype block

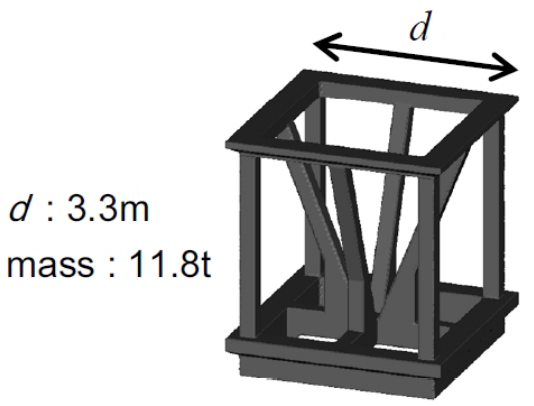

(b) Improved block

Figure 3. Shapes of the blocks.

\section{Numerical Computation Method}

Numerical computation of the falling behavior of the block in water was conducted to improve the shape of the block. An open source CFD software OpenFOAM was used. The governing equations for the fluid domain consist of the 3-dimentional incompressible Navier-Stokes equation and the 
continuity equation. For the rigid body, the equations of motion for the translation and rotation were solved. The finite volume method was used and an unstructured grid subdivided around the rigid body shown in Fig. 4 was used. At every time step, the computation of the fluid domain and the computation of the motion of the rigid body are done alternately. The computational grid of the fluid domain is deformed along with the movement of the rigid body. The flux generated by the movement of the grid is considered in the advection term. Fig. 5 shows an example of the deformation of the grid. One of the features of this method is that the grid around the rigid body could be kept fine and that the fluid domain around the rigid body could be computed with high accuracy. The method of the movement of the grid is detailed in Jasak and Tukovic (2007). The procedure of the computation is as follows:

1.Compute the fluid domain (solve the Navier-Stokes equation and the continuity equation).

2.Compute the force and torque acting on the rigid body.

3.Compute the motion of the rigid body (solve the equation of motion).

4.Move the boundary of the rigid body and the computational grid.

5.Move to the next time step.

6.Repeat $1-5$.

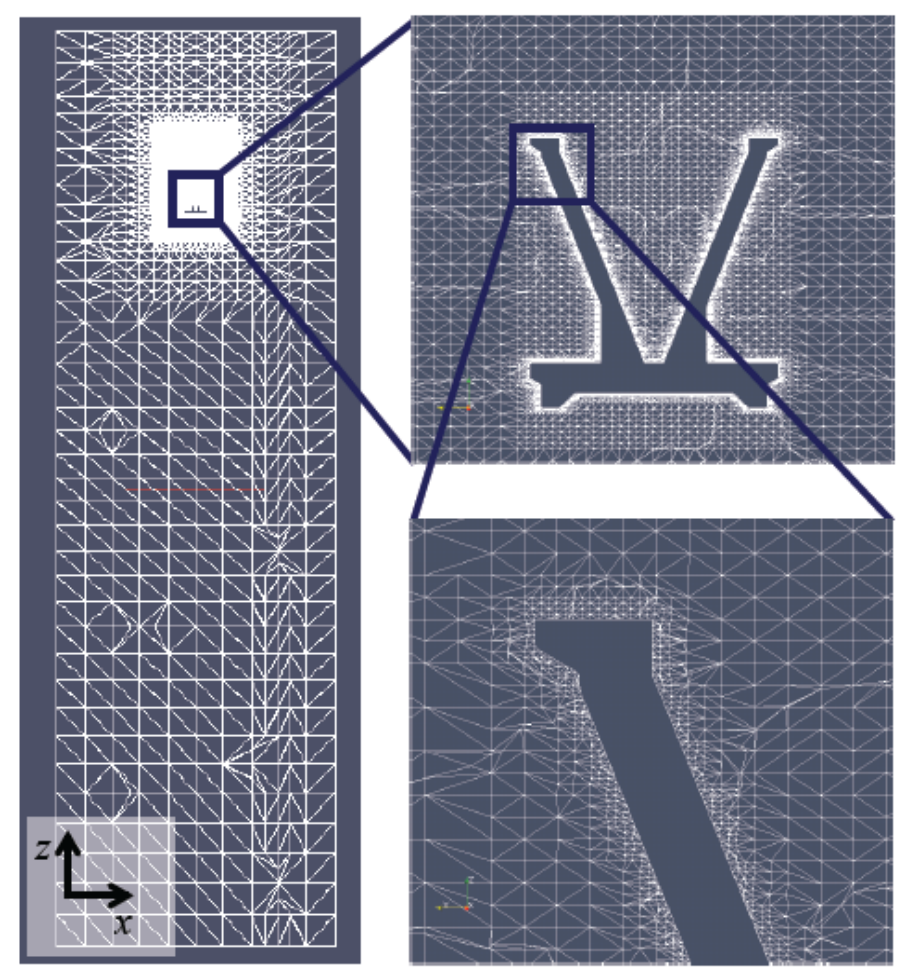

Figure 4. Sectional view of the computational grid of the "Improved block". 

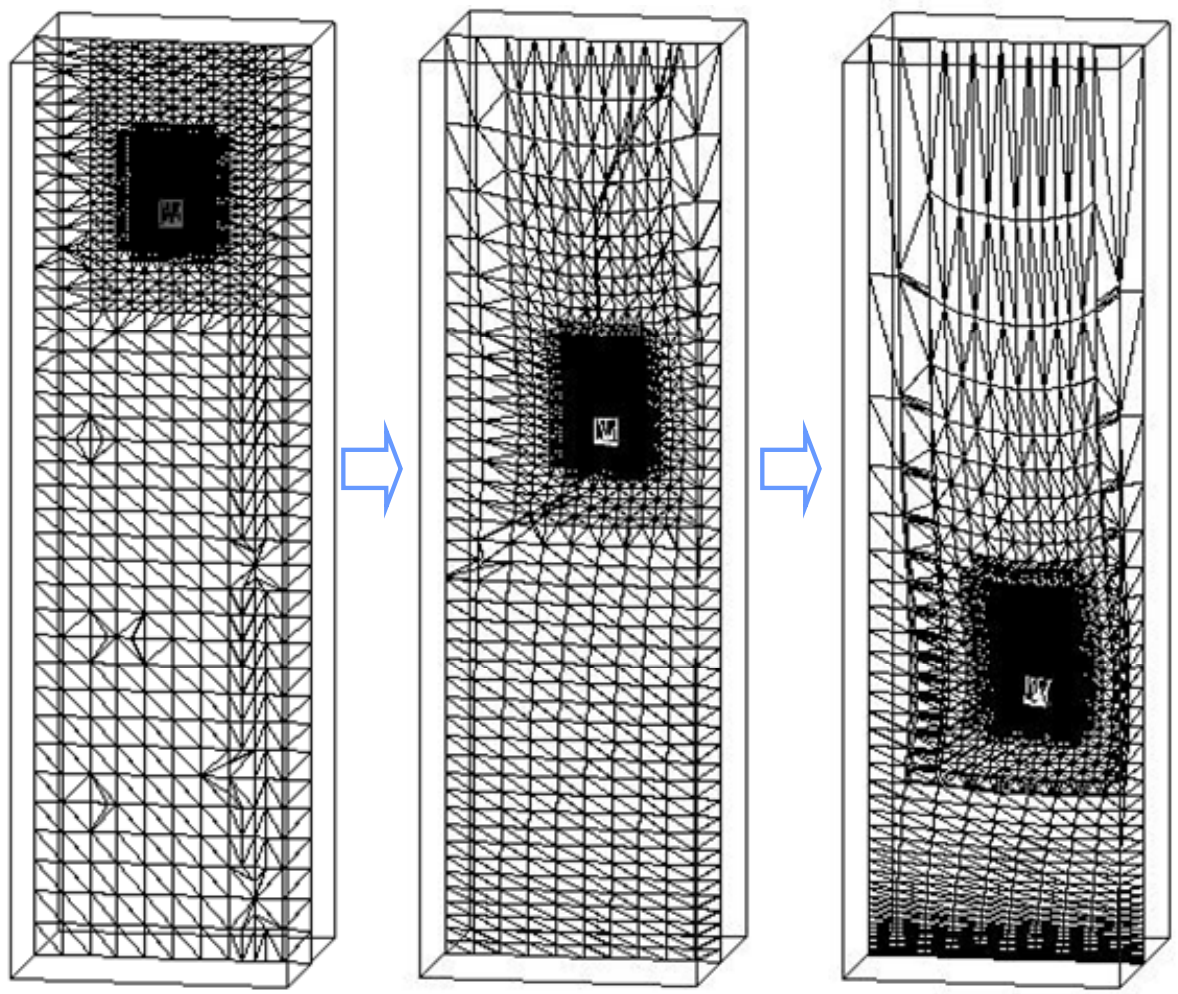

Figure 5. Example of the deformation of the computational grid.

Computation was conducted with the "Prototype block" and the "Improved block". The "Improved block" was designed based on the "Prototype block" and has an extra shelf area on the upper and lower frames attached to act as a stabilizer. The conditions of the computation are summarized in Table 1. As an initial condition, an angular velocity of $1.0 \mathrm{rad} / \mathrm{s}$ in the clockwise direction viewed from the negative direction of the $y$-axis was given in the block for the purpose of giving a small disturbance.

\begin{tabular}{|c|c|c|}
\hline \multicolumn{2}{|l|}{ Time step } & $\begin{array}{l}\text { Automatic } \\
\text { (maximum Courant number }=0.35 \text { ) }\end{array}$ \\
\hline \multirow{5}{*}{ Fluid } & Discretization method & Finite volume method \\
\hline & Computational grid & Unstructured collocated grid \\
\hline & Solution algorithm for N-S equation & PISO algorithm \\
\hline & Discretization scheme for advection term & $\begin{array}{l}\text { TVD scheme } \\
\text { (second-order accurate) }\end{array}$ \\
\hline & Turbulence model & - \\
\hline Rigid body & Time integration method & Runge-Kutta method \\
\hline \multirow{3}{*}{ Physical properties } & Kinematic viscosity coefficient & $10^{-6}\left[\mathrm{~m}^{2} / \mathrm{s}\right]$ \\
\hline & Mass density of water & $1000\left[\mathrm{~kg} / \mathrm{m}^{3}\right]$ \\
\hline & Mass density of the block & $2300\left[\mathrm{~kg} / \mathrm{m}^{3}\right]$ \\
\hline Boundary conditions & Surface of the block & No-slip \\
\hline \multirow{2}{*}{ Initial conditions } & Velocity of the block & $0[\mathrm{~m} / \mathrm{s}]$ \\
\hline & Angular velocity of the block & $\omega_{\mathrm{y}}=1.0[\mathrm{rad} / \mathrm{s}]$ \\
\hline
\end{tabular}

\section{Results of the Numerical Computation}

Fig. 6 shows a comparison of the falling behavior before and after improvement. Both computational and experimental results are shown in the figure. It can be seen that both blocks fall with a swing motion and that the amplitude of the swing motion and the rotation angle of the "Improved block" has been reduced compared to the "Prototype block" due to the extra shelf area. Although the numerical computation predicts the amplitude of the swing motion excessively compared to the experimental results with regard to the "Prototype block", the computational result of the 
"Improved block" agreed well with the experimental one. Fig. 7 shows the settling velocity of the "Improved block". The value of the settling velocity in the experiment means the average velocity obtained from the time taken to pass through each interval of $0.2 \mathrm{~m}$ in the vertical direction. It was found that the computational result shows good agreement with the experimental one in terms of terminal velocity. The water depth which reaches the terminal velocity also agrees with the experimental one.

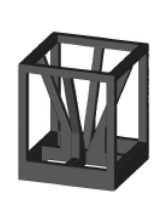

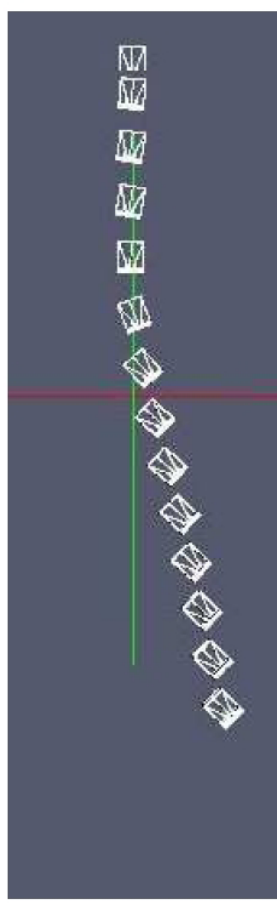

Computation

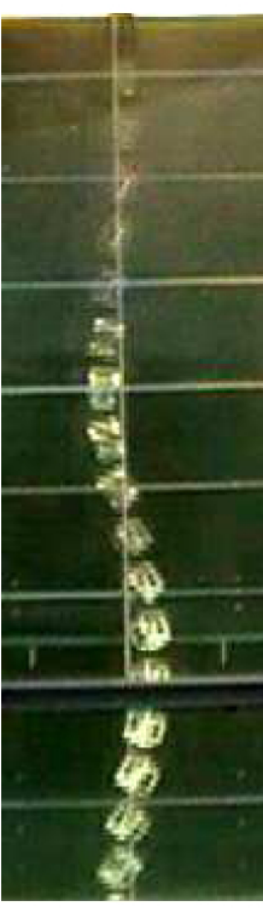

Experiment
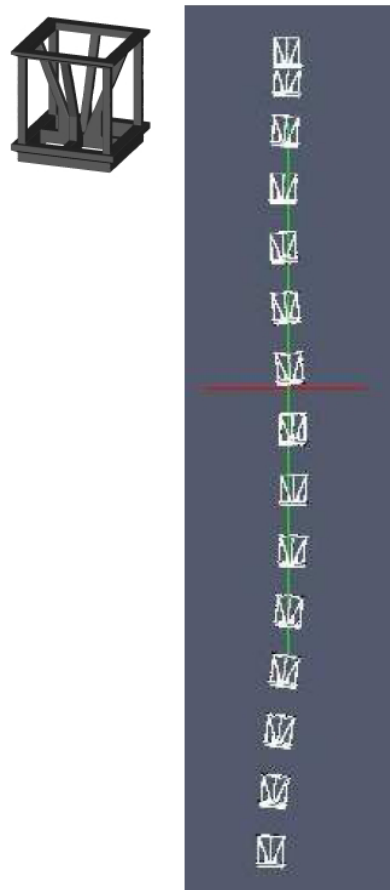

Computation

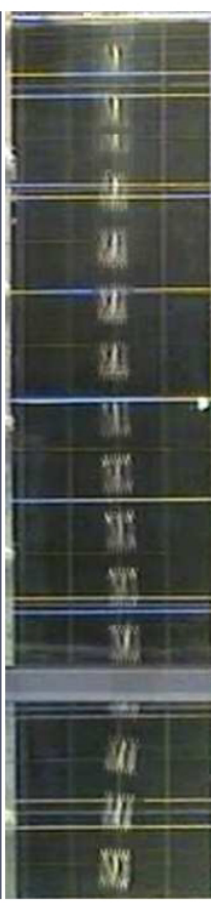

Experiment

(a) Prototype block

Figure 6. Comparison of falling behavior before and after improvement. (The time interval of display is $0.2 \mathrm{~s}$ in model scale)

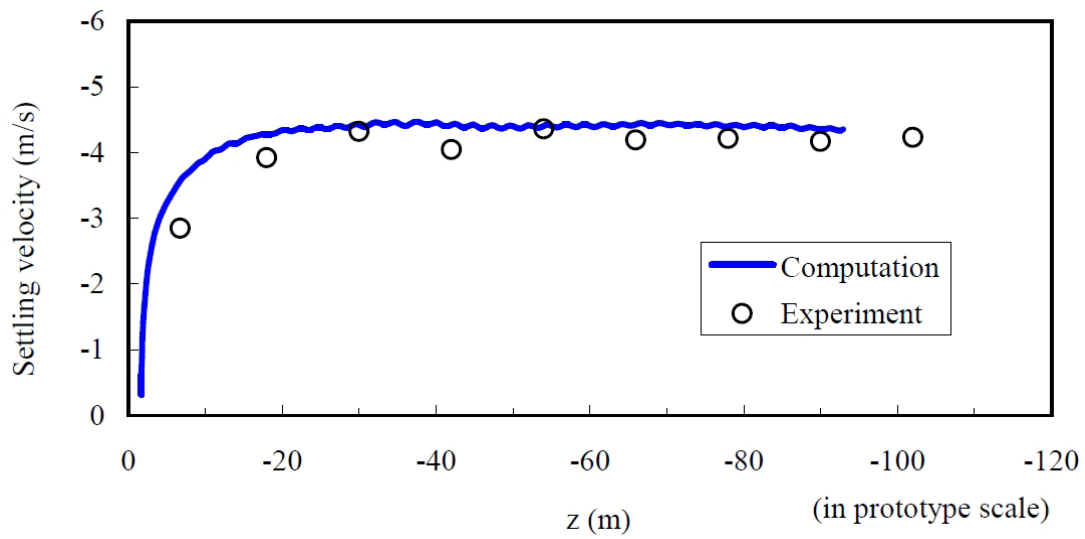

Figure 7. Settling velocity of the "Improved block".

(Experimental values show the mean velocities of each $12 \mathrm{~m}$ section)

\section{Distribution Range of the Placement Position}

The distribution range of the placement position of the "Improved block" was investigated by using a stochastic model. In this study, the stochastic model for spatial geometry of a discharged rubble 
mound given by Matsumi and Kimura (1992) was applied. The model assumes that the horizontal displacement of the settling block in a certain vertical section follows the same Gaussian distribution independent of the vertical position. The model also assumes the Markov chain theory with respect to the horizontal displacement in a certain section. That is, the next state depends only on the current state and not on the sequence of events that preceded it.

At first, the probabilistic distribution of the horizontal displacement $d x$ in a certain vertical section was investigated by analyzing the experimental data of the "Improved block". Fig. 8 shows the standard deviation of the displacement in each section. The length of each vertical section was $0.2 \mathrm{~m}$ ( $12 \mathrm{~m}$ in prototype scale). In the figure, the standard deviation is expressed in the dimensionless value by dividing by the length of the block, which is $5.5 \mathrm{~cm}$ ( $3.3 \mathrm{~m}$ in prototype scale). It can be considered that the standard deviation is almost constant independent of the vertical position. Also it is found that the influence of the flow field is small as the mean value of the standard deviation in the flow field experiments is 0.101 , which is slightly smaller than that in the still water experiments, at 0.131 .

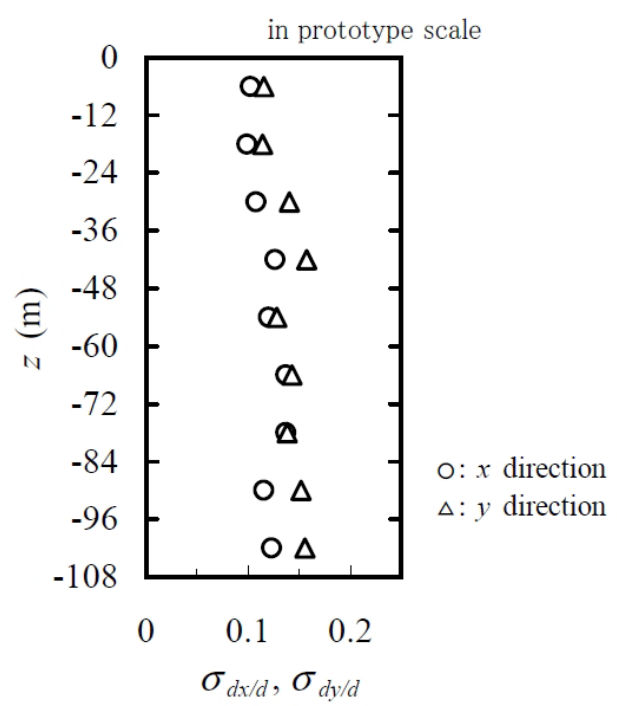

(a) in a still water

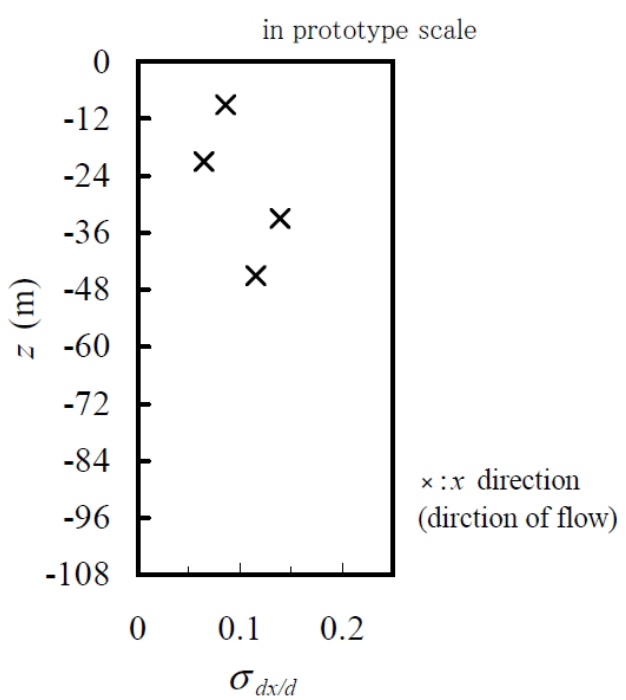

(b) in a flow field

Figure 8. Standard deviation of the horizontal displacement $d x$ in each vertical section.

Secondly, the Gaussian distribution was fitted to the distribution of the horizontal displacement $d x$ of the still water experiments to confirm the suitability. Fig. 9 shows examples of the results. The mean value of the Gaussian distribution was set to zero and the standard deviation was set to the mean value in the still water experiments which was 0.131. It can be seen that the Gaussian distribution fits well to the experimental results. 

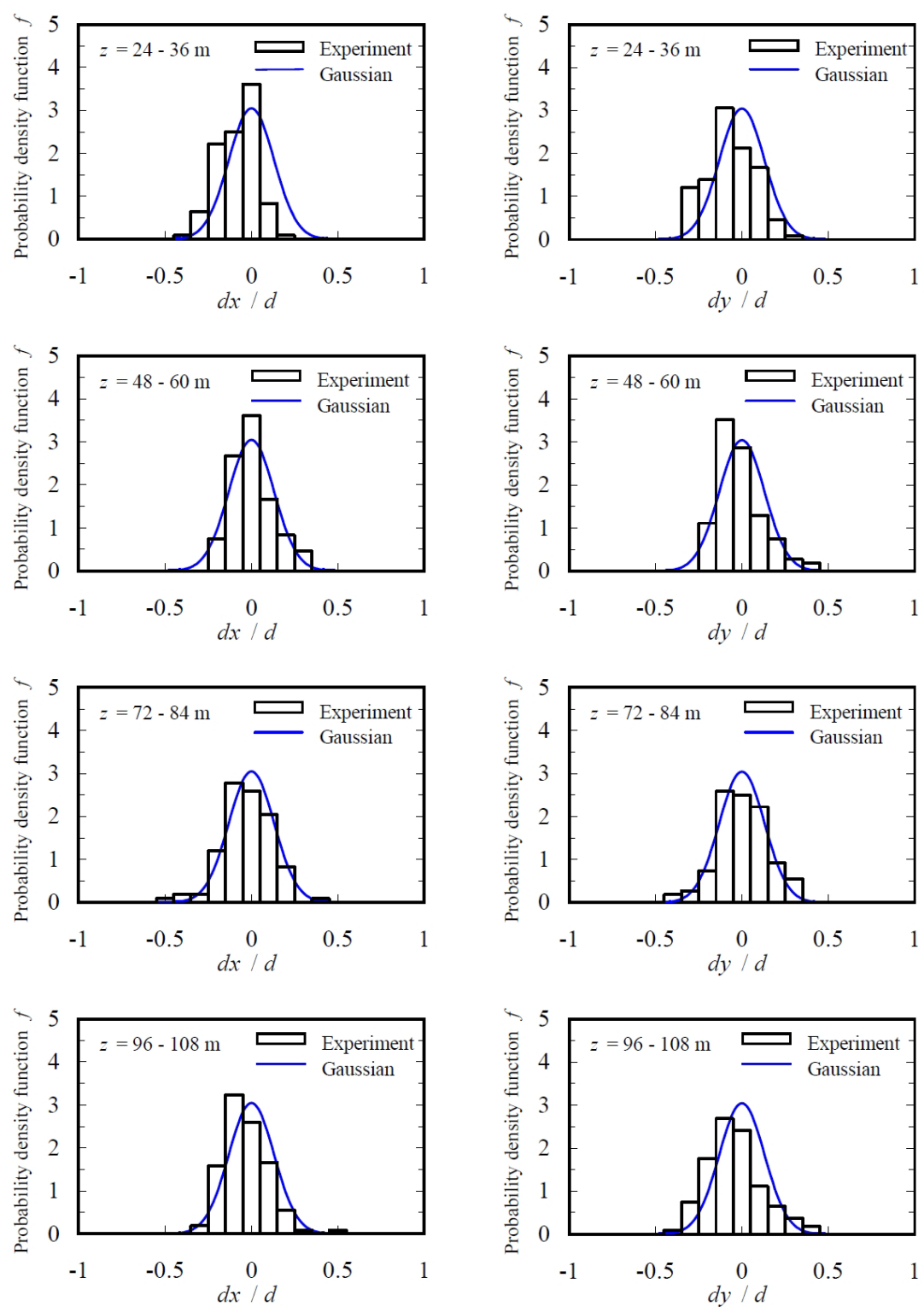

Figure 9. Relative frequency distribution of the horizontal displacement $d x$ and the Gaussian distribution.

Fig. 10 shows the predicted distribution of the horizontal position of the "Improved block" compared with the experimental results. The Gaussian distribution with zero mean value and standard deviation of 0.131 was used in the model. It can be seen that the predicted results reproduce how the distribution spread as the blocks fall deeper. By using the predicted result at the water depth of $252 \mathrm{~m}$, the range of $99 \%$ of non-exceedance probability is calculated to be $-5.1 \mathrm{~m}$ to $+5.1 \mathrm{~m}$. Since the accuracy requested for the placement is a range within $30 \mathrm{~m}$ in radius, it can be concluded that the "Improved block" can be placed with sufficient accuracy. 

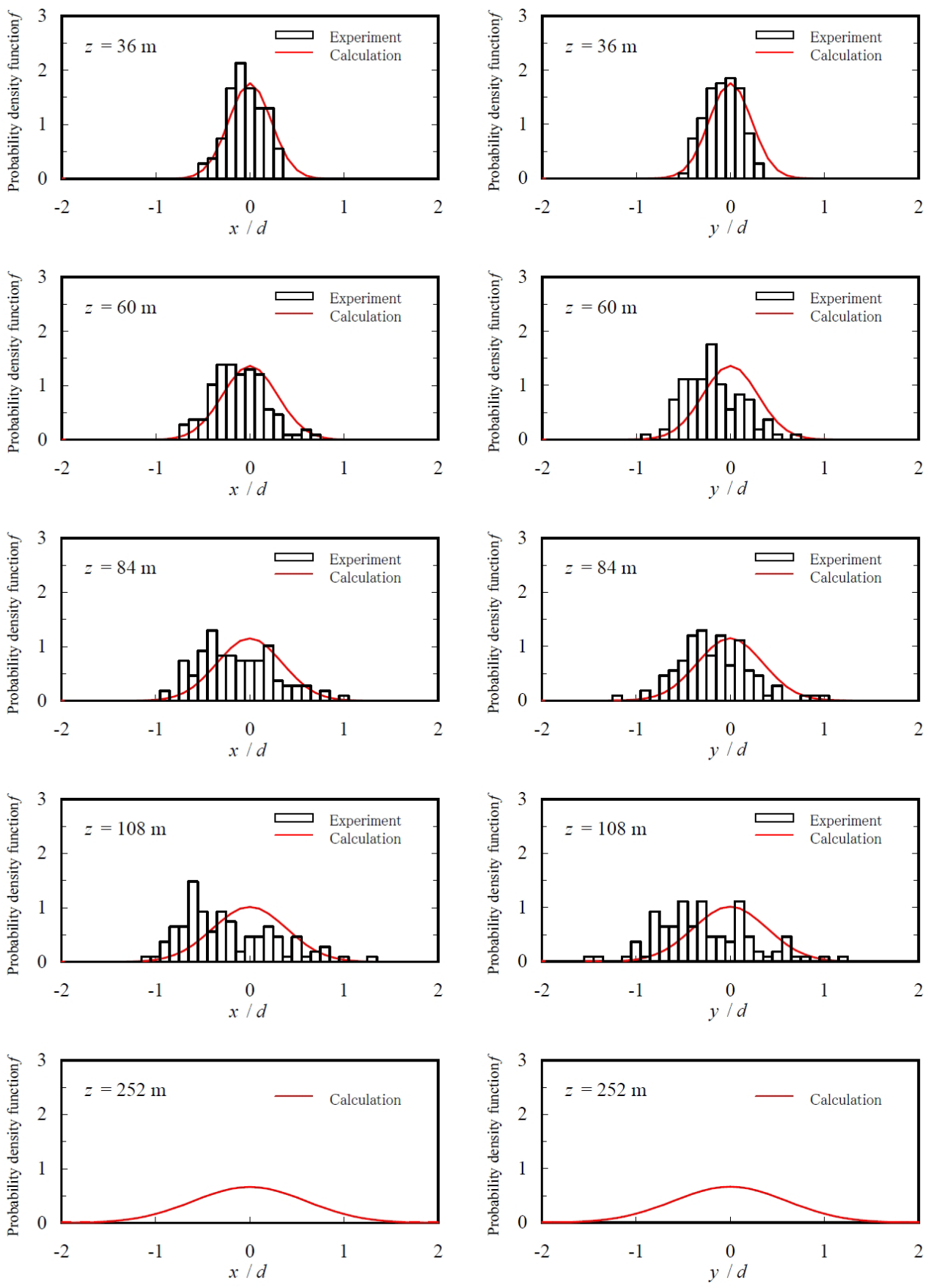

Figure 10. Probabilistic distribution of the horizontal position of the "Improved block".

\section{DEVELOPMENT OF A QUICK ESTIMATION METHOD FOR THE PLACEMENT POSITION}

\section{Basic Concept}

In actual construction, it is necessary to forecast the placement position in a short time because the release point would be determined on the floating crane taking the flow field in the site into account. The computational method which solves the Navier-Stokes equation mentioned above is not practical to forecast on site since it takes a long time to compute. Therefore, a quick estimation method was developed.

To reduce the computational time, the hydrodynamic force acting on the block was evaluated using the hydrodynamic force coefficients instead of computing the pressure field by solving the 
Navier-Stokes equation. In addition, the rotational motion of the block was ignored because the effect of the rotational motion to the average falling track would be small.

\section{Quick Estimation Method}

Fig. 11(a) shows the coordinate system for the calculation. Kumagai et al. (2004) proposed a prediction method for a falling behavior of a block for the construction of an artificial mound for upwelling generation. In their method, the hydrodynamic force acting on the block is evaluated by using drag coefficient. Then the displacement of the block is obtained by solving the equation of motion. In this study, not only the drag force but also the forces in two other directions orthogonal to each other are considered by using three hydrodynamic force coefficients to enhance the accuracy. The equation of motion is shown as follows:

$$
\left(M+\rho_{w} k_{m} V\right) \frac{d \boldsymbol{v}_{b}}{d t}=\boldsymbol{F}+\left(M-\rho_{w} V\right) \boldsymbol{g}
$$

where, $M:$ the mass of the block, $\rho_{w}:$ the density of water, $k_{m}:$ the added mass coefficient, $V:$ the volume of the block, $\boldsymbol{F}:$ the hydrodynamic force. $\boldsymbol{F}$ is expressed as follows:

$$
\begin{gathered}
\boldsymbol{F}=\frac{1}{2} C_{D} \rho_{w} A\left|\boldsymbol{v}_{r}\right|^{2} \boldsymbol{l}+\frac{1}{2} C_{L} \rho_{w} A\left|\boldsymbol{v}_{r}\right|^{2} \boldsymbol{m}+\frac{1}{2} C_{S} \rho_{w} A\left|\boldsymbol{v}_{r}\right|^{2} \boldsymbol{n} \\
\boldsymbol{v}_{r}=\boldsymbol{v}_{w}-\boldsymbol{v}_{b}
\end{gathered}
$$

where, $C_{D}, C_{L}, C_{S}$ : the hydrodynamic force coefficients, $v_{w}$ : the flow velocity, $v_{r}$ : the relative flow velocity viewed from the block (fig. 11(b)), $A$ : the representative area of the block, which was defined as the projected area viewed from $z$ axis. $\boldsymbol{l}, \boldsymbol{m}, \boldsymbol{n}$ are the unit vectors orthogonal to each other. $\boldsymbol{l}$ was defined as the vector of the same direction to the relative flow velocity, $\boldsymbol{m}$ was defined as the vector perpendicular to $\boldsymbol{l}$ and $\boldsymbol{n}, \boldsymbol{n}$ was defined as the vector perpendicular to the relative flow vector and $z$ axis. Therefore, $\boldsymbol{l}, \boldsymbol{m}, \boldsymbol{n}$ are expressed as follows:

$$
\begin{gathered}
\boldsymbol{l}=\frac{\boldsymbol{v}_{r}}{\left|\boldsymbol{v}_{r}\right|} \\
\boldsymbol{m}=\frac{\left(\boldsymbol{k} \times \boldsymbol{v}_{r}\right) \times \boldsymbol{v}_{r}}{\left|\left(\boldsymbol{k} \times \boldsymbol{v}_{r}\right) \times \boldsymbol{v}_{r}\right|} \\
\boldsymbol{n}=\frac{\boldsymbol{k} \times \boldsymbol{v}_{r}}{\left|\boldsymbol{k} \times \boldsymbol{v}_{r}\right|}
\end{gathered}
$$

where, $\boldsymbol{k}$ is the unit vector in the direction of the $z$ axis. The hydrodynamic force coefficients $C_{D}, C_{L}$, $C_{S}$ were calculated beforehand by the method which will be described in the next section. The coefficients corresponding to various angles were calculated in advance because it is considered that the values of the coefficients depend on the direction of the relative flow velocity. The direction of the relative flow velocity is expressed by two angles $\theta_{V}$ and $\theta_{H}$. $\theta_{V}$ was defined as the angle formed by the relative flow velocity and $z$ axis, $\theta_{H}$ was defined as the one formed by $x$ axis and the horizontal component of the relative velocity as shown in Fig. 11(c).

The equation of motion (Eq. 1) is solved by using the Newmark- $\beta$ method which is one of the implicit numerical integration methods. When solving Eq. 1, the values of $C_{D}, C_{L}, C_{S}$ are updated at every time step depending on the direction of the relative flow velocity $\boldsymbol{v}_{r}$. 


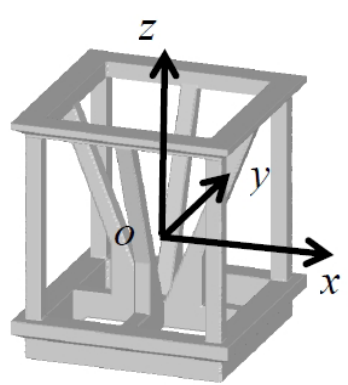

(a) Coordinate system

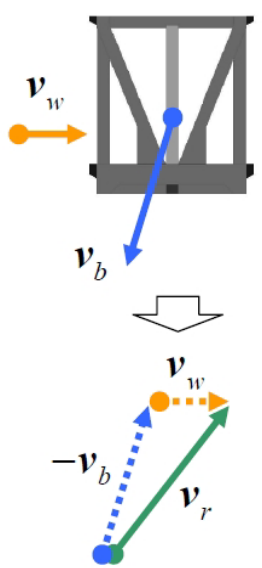

(b) Relative flow velocity

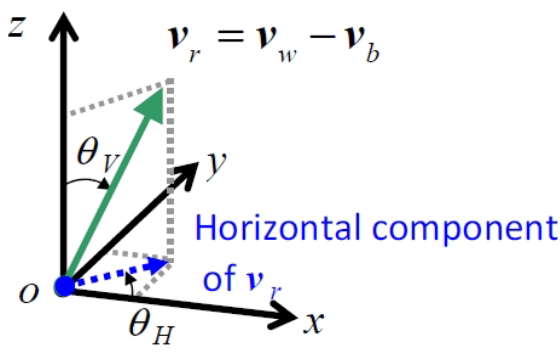

(c) Direction of the relative flow velocity

Figure 11. Definition of the coordinate system, the relative flow velocity, and the direction of the relative flow velocity.

\section{Computation of the Hydrodynamic Force Coefficients}

The hydrodynamic force coefficients were obtained by computing the hydrodynamic force acting on a block fixed in a steady flow. As shown in Fig. 12, the block was placed at the center of the computational domain of $0.6 \mathrm{~m} \times 0.6 \mathrm{~m} \times 0.6 \mathrm{~m}$. A uniform flow of $1.0 \mathrm{~m} / \mathrm{s}$ was given from the lower boundary of the computational domain. Computation was performed with varying the angle of the block. A steady-state incompressible flow solver in OpenFOAM was used. Table 2 shows the conditions of the computation. Fig. 13 shows the computed coefficients of the "Improved block". The coefficients used in the computation of the falling track can be obtained by linear interpolation of the values shown in the figure.

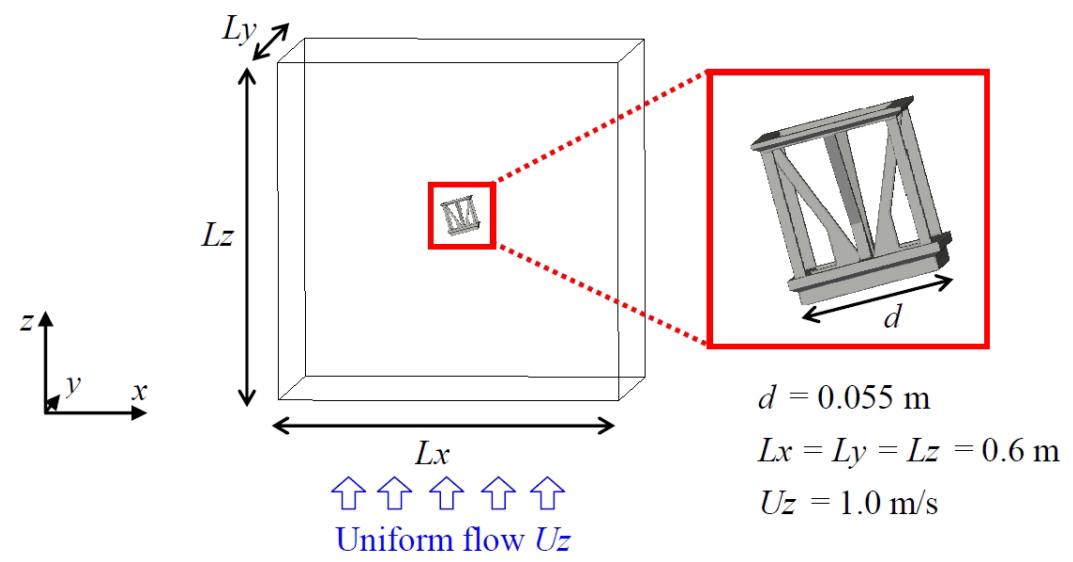

Figure 12. Computational domain for the computation of the hydrodynamic force coefficients. 


\begin{tabular}{|c|c|c|}
\hline \multicolumn{2}{|l|}{ Discretization method } & Finite Volume method \\
\hline \multicolumn{2}{|l|}{ Computational grid } & Unstructured collocated grid \\
\hline \multicolumn{2}{|c|}{ Solution algorithm for $\mathrm{N}-\mathrm{S}$ equation } & SIMPLE algorithm \\
\hline \multicolumn{2}{|c|}{ Discretization scheme for advection term } & $\begin{array}{l}\text { TVD scheme } \\
\text { (second-order accurate) }\end{array}$ \\
\hline \multicolumn{2}{|l|}{ Turbulence model } & - \\
\hline \multirow{2}{*}{ Physical properties } & Mass density of water & $1000\left[\mathrm{~kg} / \mathrm{m}^{3}\right]$ \\
\hline & Kinematic viscosity coefficient & $10^{-6}\left[\mathrm{~m}^{2} / \mathrm{s}\right]$ \\
\hline \multirow{4}{*}{ Boundary conditions } & Surface of the block & No-slip \\
\hline & Lateral boundary & Slip \\
\hline & Lower boundary & $\mathrm{Uz}: 1.0[\mathrm{~m} / \mathrm{s}], p:$ Zero normal gradient \\
\hline & Upper boundary & $\mathbf{U}:$ Zero normal gradient, $p: 0\left[\mathrm{~N} / \mathrm{m}^{2}\right]$ \\
\hline
\end{tabular}
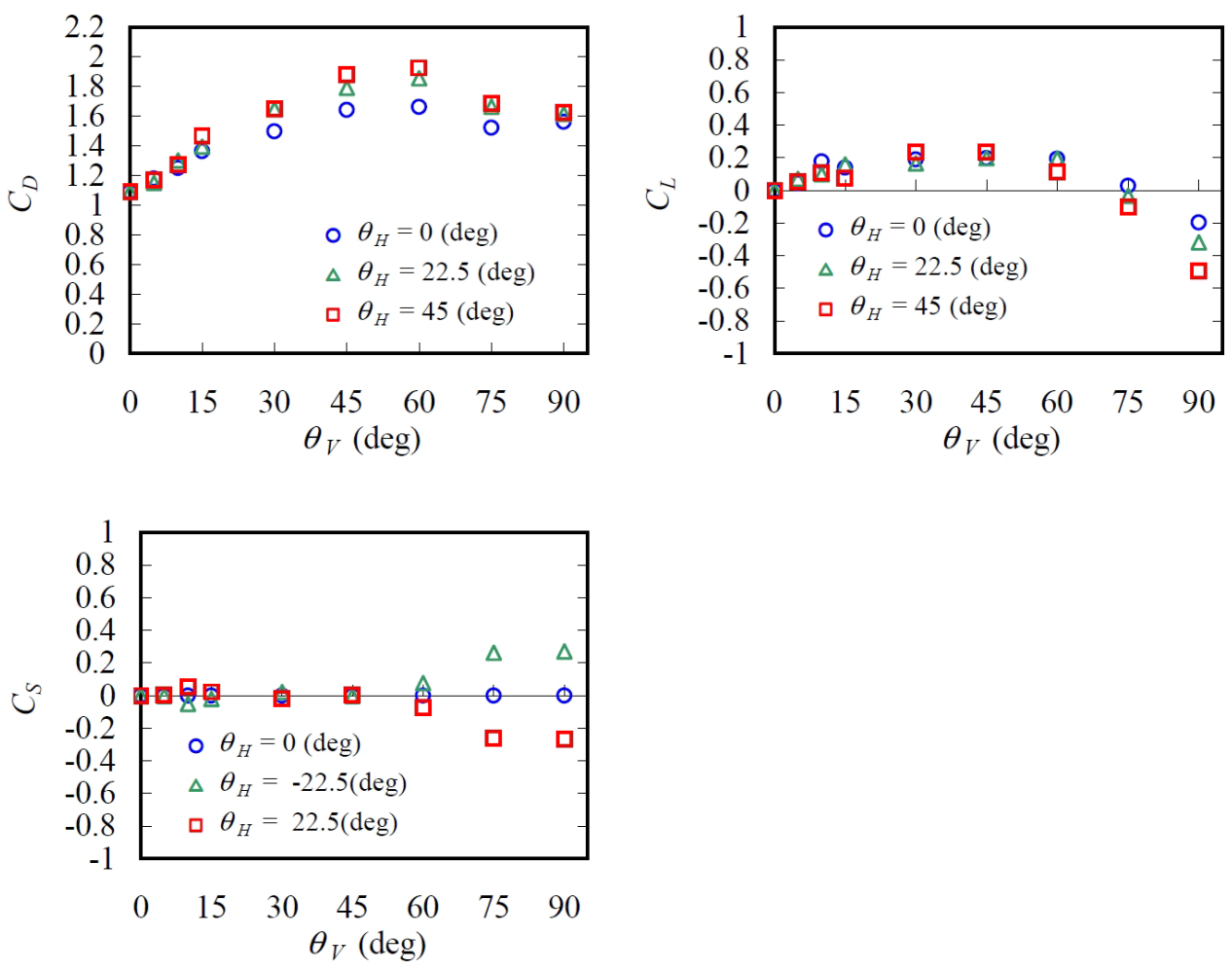

Figure 13. Hydrodynamic force coefficients of the "Improved block".

\section{Validation of the Estimation Method}

To validate the estimation method, computed falling behavior in a flow field was compared to the experimental one. The flow field was set to the same conditions as in the experiments in a flow field. That is, the flow velocity was set to $5 \mathrm{~cm} / \mathrm{s}(0.39 \mathrm{~m} / \mathrm{s}$ in prototype scale) and vertically uniform. The computation was conducted with the "Improved block". The conditions of the computation are summarized in Table 3.

\begin{tabular}{|l|l|}
\hline \multicolumn{2}{|l|}{ Table 3. Conditions of the computation of the estimation method. } \\
\hline Model scale & $1 / 60$ \\
\hline Mass of the block & $5.357 \times 10^{-2}[\mathrm{~kg}]$ \\
\hline Volume of the block & $2.329 \times 10^{-5}\left[\mathrm{~m}^{3}\right]$ \\
\hline Representative area of the block & $1.619 \times 10^{-3}\left[\mathrm{~m}^{2}\right]$ \\
\hline Time integration method & Newmark- $\beta(\beta=0.1666)$ \\
\hline Time step & $0.001[\mathrm{~s}]$ \\
\hline Mass density of water & $1000\left[\mathrm{~kg} / \mathrm{m}^{3}\right]$ \\
\hline Mass density of the block & $2300\left[\mathrm{~kg} / \mathrm{m}^{3}\right]$ \\
\hline Added mass coefficient & 1.2 \\
\hline
\end{tabular}


Fig. 14(a) shows a comparison of the falling tracks. Because the rotational motion is ignored in this method, it did not reproduce the oscillating behavior. However, it was found that this method could sufficiently predict the average falling track. Fig. 14(b) shows a comparison of the settling velocity. Although the quick estimation method predicts slightly increased speed at the beginning of the fall, the terminal velocity agreed well with the experimental and computational results. Furthermore, the computational time decreased remarkably to a few seconds. All things considered, this quick estimation method can be applied to predict the placement position on site.

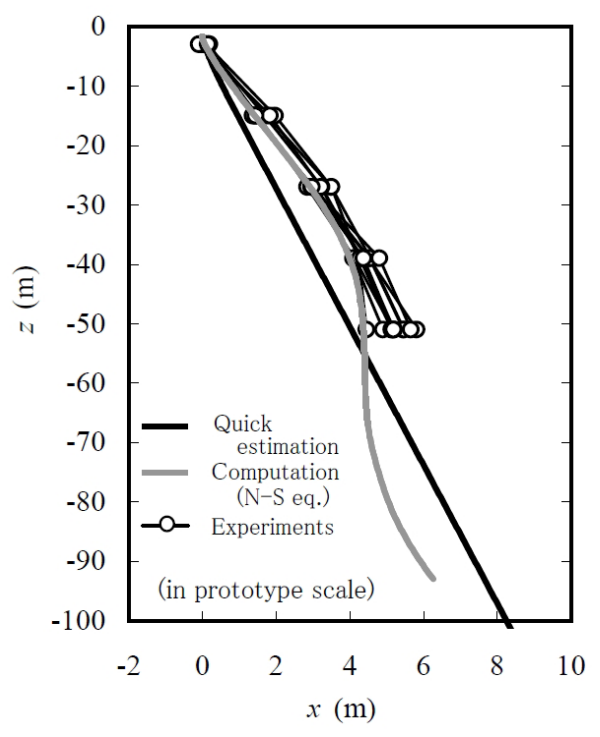

(a) Falling tracks

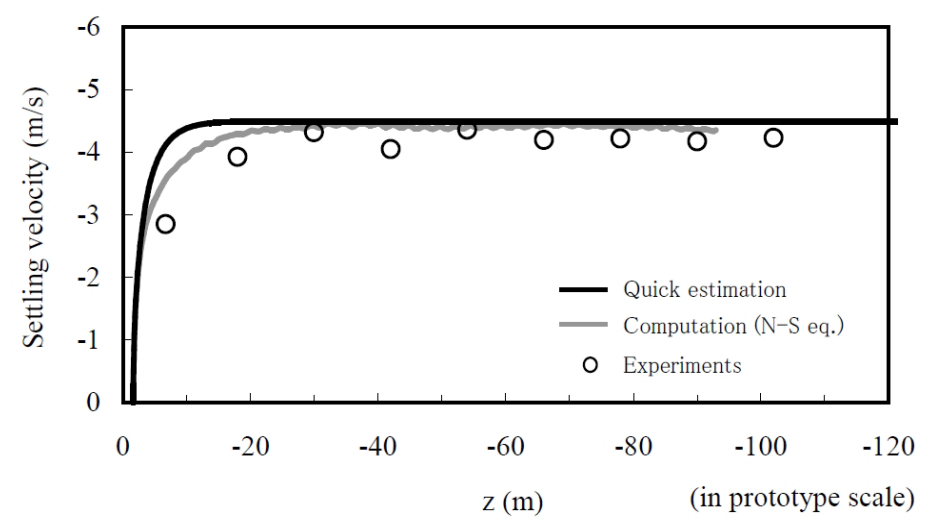

(b) Settling velocities

Figure 14. Comparison of the falling tracks and the settling velocities of the "Improved block" in a flow field.

\section{CONCLUSIONS AND FUTURE WORK}

A new fish reef block utilizing free fall in the deep sea area was developed. The block is based on a cube-shaped frame structure with an extra shelf area added on the upper and lower frames attached to act as a stabilizer. The falling behavior of the block in water was investigated by hydraulic model experiments and 3-D numerical computations. It was found that the amplitude of the swing motion and the rotation angle had been reduced due to the extra shelf area. Also, the falling behavior of the block with such a complicated shape was found to be computed accurately by the numerical computation method shown in this paper. The distribution range of the developed block on the sea bottom was predicted by stochastic model and it was proved to be sufficiently narrow.

In addition, a quick estimation method for the placement position was developed to determine the release point of the block in actual construction. By using hydrodynamic force coefficients to evaluate the forces acting on the block, the method can estimate the falling behavior of the block in a flow field 
with sufficient accuracy and in a short time. As a result, it has become possible to place fish reef blocks in deep sea areas economically and accurately.

Considering actual construction, further study is needed. For example, it is necessary to examine the falling behavior when the block is released in a state where the block is tilted, and where the block is moving at certain speeds. Also, aiming at application to more general fish reef blocks, the mechanism of falling behavior in water will be investigated by numerical analysis.

\section{ACKNOWLEDGMENTS}

The authors would like to express their gratitude to Prof. Y. Matsumi, Tottori University, for providing the program on the probability distributions and valuable advice in this study.

\section{REFERENCES}

Jasak, H. and Z. Tukovic. 2007. Automatic Mesh Motion for the Unstructured Finite Volume Method, Transactions of FAMENA, 30(2), 1-18.

Kumagai, T., S. Yamamoto, T. Hirayama, T. Manabe, K. Kobayashi, H. Shimizu, T. Kawaguchi, K. Hirata and K. Komiya. 2004. Movement of Cube Blocks Dropped from Barge Vessel to Construct Artificial Marine Ridge, Annual Journal of Coastal Engineering, JSCE, Vol.51, 801-805 (in Japanese).

Matsumi, Y. and A. Kimura. 1992. Hydraulic approach to determining optimum interval of discharge sites of barge in constructing rubble foundation of deep water breakwater, Proceedings of $23^{\text {rd }}$ International Conference on Coastal Engineering, ASCE, 3149-3162.

Moriyama, M. 2011. Effect of Protected Area on Resource of Snow Crab and Flathead Flounder, Fisheries Engineering, Vol.47, No.3, 223-225 (in Japanese). 